\title{
Simple interventions for maximum impact in primary care in the UK
}

Author: L.Edwards (Diabetes UK, Service Redesign and Improvement Consultancy)

\section{Background}

In the UK there is a problem with variations in local care at a Clinical Commissioning Group (CCG) level. The Diabetes UK Consultancy was set up to help areas tackle their varying outcomes and improve the lives of people living with diabetes in the UK. We hoped a simple, focussed and individual approach would provide a clear pathway to achieving gains in health outcomes.

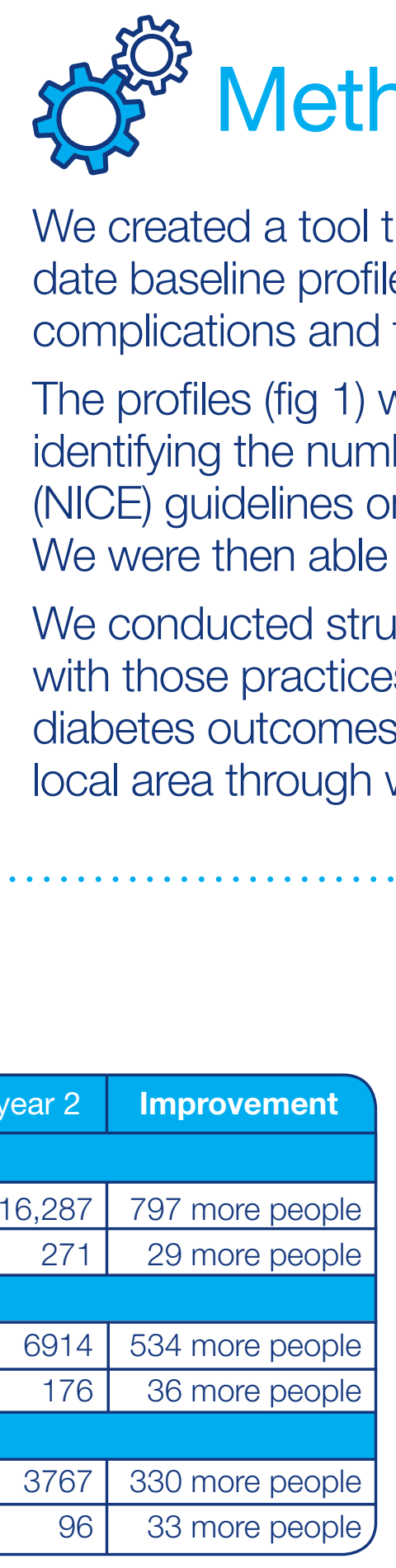

Table 1: Highlights of improvements across a CCG identified through our data collection.

The CCG has a Practice Improvement Officer who visits each practice annually. The profiling too informed and structured these discussions, playing a critical role in improving outcomes.

\begin{tabular}{|l|r|r|r|r|r|}
\cline { 2 - 6 } \multicolumn{1}{c|}{} & \multicolumn{2}{c|}{ Baseline at year 1 } & \multicolumn{2}{c|}{ Re-run at year 2} & Improvement \\
\hline Diabetes population & $5.10 \%$ & 788 & $5.04 \%$ & 796 & 8 more people \\
\hline BP checked in last 12 months & $89 \%$ & 698 & $97 \%$ & 769 & 71 more people \\
\hline Cholesterol checked in last 12 months & $84 \%$ & 664 & $94 \%$ & 748 & 84 more people \\
\hline HbA1c checked in last 12 months & $86 \%$ & 678 & $95 \%$ & 756 & 78 more people \\
\hline Missing all checks in last 12 months & $6.34 \%$ & 50 & $2.51 \%$ & 20 & 30 less people \\
\hline BP target achievement & $72.59 \%$ & 572 & $87.94 \%$ & 700 & 128 more people \\
\hline Cholesterol target achievement & $68.78 \%$ & 542 & $76.01 \%$ & 605 & 63 more people \\
\hline HbA1c target achievement & $47.08 \%$ & 371 & $53.89 \%$ & 429 & 58 more people \\
\hline All three target achievement & $32.61 \%$ & 257 & $39.70 \%$ & 316 & 59 more people \\
\hline
\end{tabular}

Table 2: Highlights of Improvement in one London practice that adopted recommendations from Best Practice Modelling.

When working with primary care we recommend that practices make two key changes. This enables the tasks to be easily implemented and achievable. This practice made the following changes:

- A new intervention to improve uptake of annual HbA1c screening.

- New follow up procedures for people who do not attend appointments.

\section{BDiscussion}

Providing primary care with accurate insights into their diabetes population and identifying simple steps to focus on for improvement instead of a more complex approach can lead to real world improvements in diabetes control and care and help avoid serious diabetes related complications.

The results within the first year of these simple improvement programmes indicates that a clear, sustainable approach delivers significant improvements in health outcomes.

The consultancy at Diabetes UK is now working with a number of partners across the length and breadth of the country to realise similar improvements in outcomes. The profiling tool is consistently highlighting aspects of care in need of improvement. Subsequently, informed strategic decisions can be made to better ensure the needs of people with diabetes are met, and costly complications that impact on quality of life are avoided.

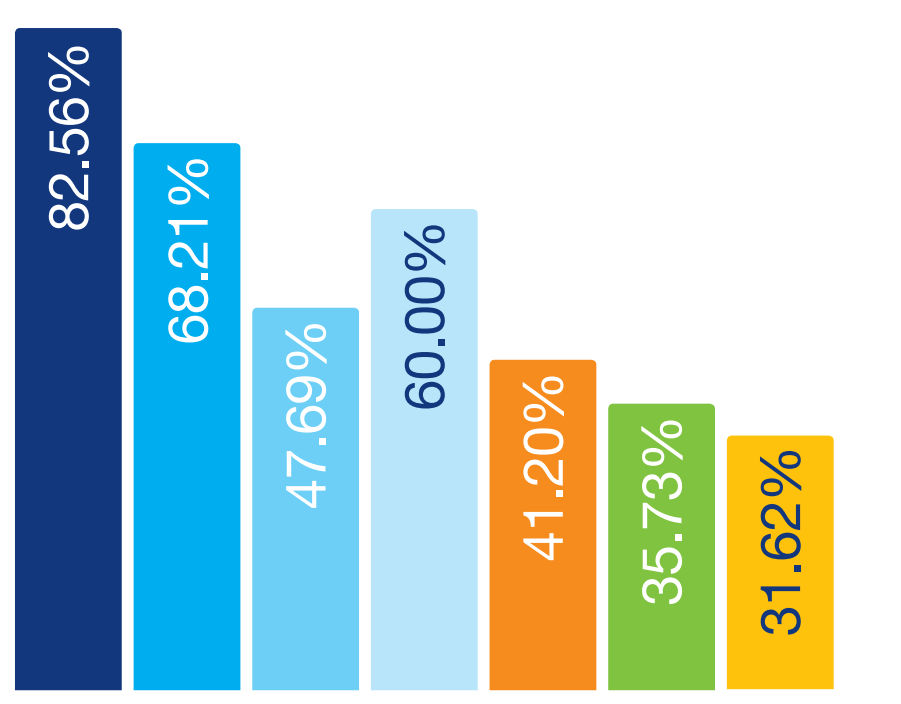

Blood pressure (<140/80)

Cholesterol $(<5)$

$\mathrm{HbA} 1 \mathrm{c}(<53)$

$\mathrm{BP}$ and cholesterol

$\mathrm{BP}$ and $\mathrm{HbA} 1 \mathrm{c}$

Cholesterol and $\mathrm{HbA} 1 \mathrm{c}$

BP / cholesterol / HbA1c (all three targets)

Fig 1: Excerpt from a Diabetes Profile, detailing treatment target achievement. 